Chapter 10

\title{
Coronary Computed Tomography Angiography
}

\author{
Stefan Baumann, Philipp Kryeziu, \\ Marlon Rutsch and Dirk Lossnitzer
}

Additional information is available at the end of the chapter

http://dx.doi.org/10.5772/67800

\begin{abstract}
Coronary computed tomographic angiography (cCTA) as a noninvasive approach underlies a rapid technological development with an impressive improvement of spatial and temporal resolution of the images. Therefore, it has become an accurate and cost-effective method to detect or exclude obstructive coronary artery disease (CAD) in patients with low to medium cardiovascular risk profile, as recommended by the ESC/AHA/ACC guidelines. The results show an excellent sensitivity, but still with a lack of specificity compared with invasive measurement. Several novel techniques like myocardial perfusion, plaque characterization or CT-based measurement of the fractional flow reserve have been developed to improve the positive predictive value and create more accurate results in detecting hemodynamically relevant stenoses. Moreover, during the last decade, the need to reduce radiation dose has become a central issue in clinical use, while the current generation of $\mathrm{CT}$ scanners has drastically lowered radiation dose. In conclusion, cCTA has become a promising alternative to invasive cardiac catheterization with still existing limitations. Thus, an appropriate patient selection is mandatory to utilize the advantages of this technique.
\end{abstract}

Keywords: coronary artery disease, coronary computed tomography angiography, coronary plaque, CT perfusion, CT-fractional flow reserve

\section{Introduction}

In the beginning of computed tomography $(\mathrm{CT})$ era, the beating heart could not be examined suitably by this technique due to its motion artefacts. While scan times and consecutively temporal resolution, enhanced rapidly it has become a more accurate noninvasive imaging method for cardiac morphology. The first attempts in using CT to visualize coronary arteries have been made in the early 1980s and were followed by the back then newly emerging 
electron beam computed tomography (EBCT), which already had scan times lower than 100 ms [1]. Clinical relevance of the coronary CT angiography (cCTA) increased distinctly with the introduction of multi detector CT (MDCT) in the late 1990s-initially with four parallel detectors, the launch of the 64-slice MDCT generation enabled CCTA to become established in routine clinical practice [2,3]. Nowadays, there are systems with up to 320-slices in clinical use, providing even lower scan times and a very high spatial resolution. Another landmark development was the introduction of the dual-source CT (DSCT) technology. DSCT contains of two tubes and detectors arranged in a $90^{\circ}$ angle, also resulting in a higher temporal resolution due to the halved rotation time. The dual-energy CT (DECT) scans allow two different tube voltages, resulting in a significant lower radiation exposure for the patient [4]. As spatial and temporal resolution achieved remarkable dimensions, recent technologic improvement emphasized particularly the reduction of radiation dose on the one hand (see Section 3.1) [5], and the expansion of cCTA on additionally functional and morphological aspects, e.g., plaque characterization, myocardial perfusion imaging, or even CT-based fractional flow reserve (CT-FFR).

\section{Coronary CT-angiography}

\subsection{Indication}

Despite its many advantages, cCTA is only one out of many clinically approved methods to examine coronary arteries. Although there are notable technical developments in evaluating functional parameters as well [6-8], the current indication is predominantly the investigation of anatomical and morphological vessel characteristics. Especially in the exclusion of coronary artery disease (CAD), cCTA plays a decisive role [9-11]. Patients presenting with symptoms of CAD and low-to-intermediate risk patients undergo rapid evaluation of their coronary arteries. To estimate the suitable method for the individual patient, pre-test riskstratification calculation plays a key role. For this purpose, Diamond-Forrester (Table 1) [12] and Genders (Table 2) [13] are well-established charts to obtain a pre-test probability of CAD based on age, sex, and chest pain constellation. However, further established cardiovascular

\begin{tabular}{lllllll}
\hline & \multicolumn{2}{l}{ Non-anginal chest pain } & \multicolumn{2}{l}{ Atypical angina } & \multicolumn{2}{l}{ Typical angina } \\
\hline Age & Men & Women & Men & Women & Men & Women \\
\hline $\mathbf{3 0 - 3 9}$ & $5.2 \pm 0.8$ & $0.8 \pm 0.3$ & $21.8 \pm 2.4$ & $4.2 \pm 1.3$ & $69.7 \pm 3.2$ & $25.8 \pm 6.6$ \\
$\mathbf{4 0 - 4 9}$ & $14.1 \pm 1.3$ & $2.8 \pm 0.7$ & $46.1 \pm 1.8$ & $13.3 \pm 2.9$ & $87.3 \pm 1.0$ & $55.2 \pm 6.5$ \\
$\mathbf{5 0 - 5 9}$ & $21.5 \pm 1.7$ & $8.4 \pm 1.2$ & $58.9 \pm 1.5$ & $32.4 \pm 3.0$ & $92.0 \pm 0.6$ & $79.4 \pm 2.4$ \\
$\mathbf{6 0 - 6 9}$ & $28.1 \pm 1.9$ & $18.6 \pm 1.9$ & $67.1 \pm 1.3$ & $54.4 \pm 2.4$ & $94.3 \pm 0.4$ & $90.6 \pm 1.0$ \\
\hline
\end{tabular}

Each value represents the percentage \pm 1 standard deviation. Adapted from Diamond et al. [12].

Table 1. Pre-test likelihood of CAD in symptomatic patients according to age and sex. 


\begin{tabular}{lllllll}
\hline & \multicolumn{2}{l}{ Non-anginal chest pain } & \multicolumn{2}{l}{ Atypical angina } & \multicolumn{2}{l}{ Typical angina } \\
\hline Age & Men & Women & Men & Women & Men & Women \\
\hline $\mathbf{3 0 - 3 9}$ & 17.7 & 5.3 & 28.9 & 9.6 & 59.1 & 27.5 \\
$\mathbf{4 0 - 4 9}$ & $\mathbf{2 4 . 8}$ & 8.0 & 38.4 & 14.0 & 68.9 & 36.7 \\
$\mathbf{5 0 - 5 9}$ & 33.6 & 11.7 & 48.9 & 20.0 & 77.3 & 47.1 \\
$\mathbf{6 0 - 6 9}$ & 43.7 & 16.9 & 59.4 & 27.7 & 83.9 & 57.7 \\
$\mathbf{7 0 - 7 9}$ & 54.4 & 23.8 & 69.2 & 37.0 & 88.9 & 67.7 \\
$\mathbf{8 0}$ & 64.6 & 32.3 & 77.5 & 47.4 & 92.5 & 76.3 \\
\hline
\end{tabular}

Adapted from Genders et al. [13].

Table 2. Updated pre-test likelihood of CAD in symptomatic patients according to age and sex.

risk factors such as smoking, dyslipidemia, hypertension, diabetes, and family history of cardiac diseases should be considered in the risk stratification as well. Depending on the individual risk constellation, cCTA may be the suitable modality in low-to-intermediate risk patients, as for high-risk patients, invasive coronary angiography remains still the gold standard, as recommended by the ESC/AHA/ACC guidelines $[9,10]$. Due to the three-dimensional visualization that can be constructed by cCTA, it can also be even used in planning and evaluating coronary artery bypass grafts (CABG) and detecting in-stent restenosis (ISR).

\subsubsection{Suspected coronary artery disease}

cCTA is excellent in visualizing coronary morphology and has emerged to an appropriate method of ruling out obstructive CAD. But by cCTA alone, the pathophysiological relevance of a detected CAD remains often unclear. Despite the remarkable advancements regarding functional parameters as for example perfusion imaging achieved by new DECT approaches, many conventional cCTAs show a rather moderate specificity regarding the functional assessment of CCTA measured stenosis. The methodical approach, as proposed by the SCCT guidelines for the interpretation and reporting of cCTA, consists of a systematic inspection of each coronary segment in multiple planes, the contemplation of image quality and artifacts and finally the evaluation of the respective lesions in regard of morphology, composition, and stenosis severity. A modified version of the well-established 1975 American Heart Association (AHA) model is used to refer to the certain segments [14]. Coronary abnormalities, plaque description or insufficient interpretability due to artifacts should be mentioned. Following this, a qualitative assessment for each segment is obtained and should be reported according to Table 3. Subsequently, a quantitative assessment of the stenosis severity is performed; the findings should be reported according to Table 4.

It has to be mentioned that these classifications are founded on morphological features only and, based on these findings, conclusions about functional or ischemic insufficiencies are not to be inferred. 


\begin{tabular}{lll}
\hline 0 & Normal & Absence of plaque and no luminal stenosis \\
1 & Minimal & Plaque with negligible impact on lumen \\
2 & Mild & Plaque with mild narrowing of the lumen \\
3 & Moderate & Plaque with moderate stenosis that may be of hemodynamic significance \\
4 & Severe & Plaque with probable flow limiting disease \\
5 & Occluded &
\end{tabular}

According to SCCT guidelines.

Table 3. Descriptors of qualitative stenosis severity.

\subsubsection{Coronary artery stent}

Due to the limited spatial resolution of the first electronic beam CT, it was initially not possible to visualize of the stented lumen and an indirect approach was applied to assess the stent patency. For this reason, contrast density was measured distally to the stent and compared with the density pattern proximal to the stented segment, in the aorta or the left ventricle, while stent patency was assumed when the contrast enhancement matched [15].

With the introduction of 64-slice scanners, a high negative predictive value could be reached for the evaluation of in-stent restenosis, while the positive predictive value is still rather worse as demonstrated by meta-analysis [16, 17]. However, there are specific technical limitations such as blooming caused by metal artifacts resulting in an underestimation of the stent lumen.

\subsubsection{Coronary artery bypass graft}

The value of cCTA in the assessment of coronary artery bypass graft (CABG) and native coronary arteries after bypass graft surgery continues to grow with advances in CT technology $[18,19]$. The improvement of spatial resolution allows the cardiovascular radiologist and cardiac surgeon to evaluate the patency of CAGB in a rapid and noninvasive manner [20]. The major advantage of cCTA over invasive angiography is the ability to simultaneously evaluate for alternate postoperative complications like malposition, kinking, or pericardial effusion.

\begin{tabular}{lll}
\hline 0 & Normal & Absence of plaque and no luminal stenosis \\
1 & Minimal & Plaque with $<25 \%$ stenosis \\
2 & Mild & $25-49 \%$ stenosis \\
3 & Moderate & $50-69 \%$ stenosis \\
4 & Severe & $70-99 \%$ stenosis \\
5 & Occluded & $100 \%$ stenosis \\
\hline
\end{tabular}

According to SCCT guidelines.

Table 4. Descriptors of quantitative stenosis severity. 


\subsection{Benefits and limitation}

The main benefit of cCTA is its noninvasive character. Although invasive coronary angiography (ICA) is an approved and secure procedure, it still involves the possibility of serious complications such as bleeding, stroke, or coronary dissection [6]. In comparison, the risks of cCTA, such as extravasation or allergic reaction to the contrast agent are less severe and common. As previously mentioned, cCTA is able to rule out CAD with excellent sensitivity and negative predictive value, both up to $99 \%$ in several studies $[9,21,22]$. Therefore, a preceding cCTA can reduce the share of unnecessarily performed ICA [11]. On the other hand, currently, the moderate specificity of cCTA causes a following ICA to validate the findings [9]. Recent developments seek to solve this issue. Further limitations result from technical conditions of computed tomography:

Although the temporal resolution has achieved levels below $80 \mathrm{~ms}$, it is still necessary for the patient to maintain a heart frequency under 70 beats per minute to obtain a sufficient image quality. This might be accomplished using beta-blockers, but not all patients are suitable for auxiliary agents. Regarding patients who are unable to follow breathing orders, but especially patients with cardiac arrhythmias, prospectively electrocardiogram (ECG)-triggered images are prone to artifacts. New approaches in ECG triggering seek to react flexibly to arrhythmia but have to be implemented in the clinical routine. Retrospectively ECG-gated image acquisition is less interference-prone, but is along going with higher radiation doses. ECG-dependent dose reduction is required. Furthermore, a high coronary calcification or iatrogenic metallic material may lead to so-called blooming or streak artifacts, which tend to over-estimate the severity of stenoses [23, 24]. A better temporal resolution, acquired e.g., by using DSCT allows reduction of blooming artifacts. Radiation dose represents another important disbenefit of cCTA, which is explained later in detail.

\section{Technical development}

\subsection{Radiation dose}

Since its introduction into clinical use, a constantly mentioned point of criticism of cCTA is the radiation the patient is exposed to. While referring to this topic, one should distinguish the terms "radiation exposure," which describes the radiation emitted by the X-ray source, and "radiation dose," which indicates the amount of radiation absorbed by the patient [25]. The early concerns were not unjustified, as the novel scanners with 16 or 64 slices showed radiation doses above $10 \mathrm{mSv}$, even up to $21 \mathrm{mSv}$ [26, 27], and radiation resulting of CT examinations make up a large share of the populations radiation exposure [28]. But subsequently, a substantial reduction of the applied radiation doses was achieved by different approaches: cCTA images are usually acquired using retrospective ECG gating, which requires a lower pitch and a longer duration, resulting in higher doses than prospective ECG triggering. Dose reduction is acceived using ECG gating or implementation of suitable ECG-triggering protocols. The first option is realized through ECG-dependent tube current modulation. The best image quality is obtained in the late-diastolic phase of the heart cycle; therefore, the tube 
current can be decreased in the remaining phase, resulting in a radiation dose lowered up to $50 \%[29,30]$. Under certain circumstances, it is possible to perform cCTA by prospective ECG triggering and sequential scanning. Patients with a low and stable heart rhythm and without an indication for functional testing are qualified for this technique in line with SCCT guidelines [31]. This attempt could reduce the radiation dose to $70-80 \%[29,32]$. Both options are optimal if either a scanner with 256 or more slices or a DSCT is used. Furthermore, use of DSCT enables further decrease due to its higher pitch rates at higher heart rates, since multisegment reconstruction is not necessary $[33,34]$. Additional reduction is accomplished by a tube voltage of $100 \mathrm{kV}$ or even $80 \mathrm{kV}$ instead of the usual $120 \mathrm{kV}$, which can be performed depending on the patient's body mass $[30,31]$. The image postprocessing technique of iterative reconstruction (chapter 3.2) also contributes to reduction of radiation dose. With all these measures taken into consideration, cCTA reached radiation doses lower than $4 \mathrm{mSv}$, therefore being in the range of the average yearly background radiation dose, in certain conditions even in submillisievert range $[35,36]$.

\subsection{Image reconstruction}

Nowadays, two methods of image reconstruction are in use, analytical filtered back projection (FBP) and iterative reconstruction (IR). The initially used technique was indeed the more complex IR [37], but soon its use was limited by the computational power of erstwhile processors. The method was displaced by FBP, which still is the most widely used technique nowadays.

In FBP, the measured intensity is described as an integral function, and the reconstruction data is obtained through solution of the resulting equations, which is called back projection. Additionally, a filter component compensates low-pass signals. If a higher spatial resolution is required, the filter can be adjusted accordingly. However, this adaptation of the filter causes a higher image noise, since image sharpness and image noise are proportional [38].

IR seeks to solve this problem, and since nowadays, not only CT hardware but also software underwent enormous advances, complex computational operations are more and more available. Iterative reconstruction accomplishes the back projection through the comparison of two components; a simulated first image estimation on the one hand and the actual measured projection on the other hand. Both images are automatically compared and, in case of discrepancy, the estimation is altered and another comparison is made until a default condition is achieved [38]. The underlying complex mathematical algorithms are propriety of the respective companies. Not only was IR able to break the correlation between image noise and spatial resolution, but it does so while simultaneously reducing the applied radiation dose up to $40-70 \%$, while maintaining or even increasing subjective image quality and diagnostic accuracy [39-42].

\section{Plaque characterization}

The first attempts in evaluating atherosclerotic plaques via CT have already been made 1985 [43], but this approach did not gain acceptance due to insufficient resolution and image quality. Nowadays, with a spatial resolution up to $400 \mu \mathrm{m}$, noninvasive detection and characterization 
of atherosclerotic lesion and plaque characteristics can be performed by current CT scanners. Although intravascular ultrasound (IVUS) and optical coherence tomography (OCT) provide even higher spatial resolutions up to 80 and $20 \mu \mathrm{m}$, respectively [44], and therefore are the reference standard, cCTA yields the advantage of its noninvasive character. This technique enables an evaluation and characterization of the individual plaque extent and composition in patients without the clear indication for invasive measures. Recent studies have shown the ability of cCTA to perform on a high level in comparison with earlier mentioned reference standards, thus making cCTA a promising noninvasive method in identifying high-risk atherosclerotic coronary plaques [45-47]. Plaque characterization is essential in risk stratification in patients with suspected or diagnosed CAD or ACS, hereby it is important to distinguish the terms "stable" and "vulnerable" plaque (Figure 2). The hazard in stable plaques, consisting mainly of calcifications, lies in their subsequent obstruction of vessel lumen, associated with hemodynamic insufficiency, whereas vulnerable plaques tend to rupture and can lead to occlusion of the affected vessel through the thrombogenic lesion [48]. The finding that major adverse cardiac events (MACEs) are a consequence of the hemodynamically insignificant vulnerable plaques in more of two-thirds has been already made in the end of the last century $[49,50]$, but only now it is possible to detect morphological correlates in vivo via noninvasive methods [51]. Certain morphological plaque features correlate with the presence of ruptureprone plaques, and it is yet to be examined, which of these are reliable markers of plaque vulnerability $[47,52]$. Although cCTA can distinguish distinctly between calcified, noncalcified (lipid rich/fibrotic) and mixed plaques, direct visualization of thin-cap fibroatheroma (TCFA) is currently only possible via OCT. To make plaque characterization via CCTA less dependent on the examiner's experience, scoring systems [53] and semiautomated software are ready to be implemented in clinical use, increasing operator convenience of this promising method.

\section{CT myocardial perfusion}

Due to high sensitivity and negative predictive value [54, 55], cCTA is at present an accepted diagnostic tool in detecting CAD in patients with low pretest probability [9]. However, the major limitation of CCTA remains in its low specificity and positive predictive value and the missing correlation of detected lesions and their physiological significance [56-58].

Challenge for novel diagnostic methods is to provide data about the anatomical and functional assessment of coronary stenosis. Myocardial perfusion derived from computed tomography (CTMP) is a recent instrument in diagnosis of ischemia. Compared to other functional tests, CTMP offers the substantial advantage that it is performed during ordinary CCTA. CTMP is a "one-stop shop" approach to close the gap between anatomical and functional assessment within a single imaging and could additionally limit false-positive results of cCTA [6].

Underlying principles of CTMP is the distribution and enhancement of iodinated contrast agent within the myocardium. The iodinated contrast agent is used as an indicator for myocardial blood flow and myocardial blood volume, based on the principles of the indicator-dilution theory. Myocardial areas with reduced amounts of contrast agent are indicating perfusion defects [59]. 


\subsection{Image acquisition and protocols}

Like other functional imaging methods, ordinary acquisition of CTMP consists of three sequences: a rest acquisition, an acquisition under pharmacological stress, and an acquisition of late enhancement. This approach is used to evaluate the reversibility of the ischemia [6].

Adenosine is used during the pharmacological stress acquisition for dilation of the coronary arteries with a dose ratio of $140 \mu \mathrm{g} \mathrm{kg}^{-1} \mathrm{~min}^{-1}$. This leads to a decrease of the perfusion pressure. However, compensatory dilatation of obstructed arteries is limited. Reversible ischemia is the result of decreased perfusion reserves within these vessels. This pathophysiological phenomenon is called the "steal-effect." After 2-3 min of continuous administration of adenosine with monitoring of ECG, pulse oximetry, and blood pressure, iodinated contrast agent is injected and image acquisition starts [6]. Beyond the application of iodinated contrast agent during rest and stress acquisition and adenosine during stress acquisition, beta blockers, and nitrates were administered immediately before the examination to avoid motion artifacts and to improve image quality [59]. Contraindication (e.g., contrast agent allergy, severe COPD, severe aortic valve stenosis) should be taken into consideration regarding suitability of the patient. After 5-10 min of administration of contrast agent, a delayed acquisition can provide information about nonviable myocardium [6]. Myocardial areas of ischemia or infarction are described based on the American Heart Association segmental model [14].

Regarding comparability of studies and deeper understanding, it should be noted that there is a static myocardial blood pool imaging method during first pass and apart from it a dynamic myocardial perfusion method over several time points of myocardial iodine distribution. Development in computed tomography offers with dual-energy CT a further static perfusion method. For example, differences between these techniques apply on the direct assessment of quantitative perfusion parameters or radiation exposure $[6,60]$.

\subsection{Radiation exposure}

Radiation dose of a comprehensive protocol containing rest, stress, delayed enhancement, and calcium scoring have generally been reported in the range of $12-14 \mathrm{mSv}$. This is comparable to the radiation dose during SPECT examination [6]. Modified protocols in research contain considerably lower radiation. Feuchtner et al. achieved high accuracy (sensitivity 96\%, specificity 88\%, PPV 93\%, and NPV 94\%) in a stress approach and reported radiation dose of $2.5 \mathrm{mSv}$ for cCTA and perfusion imaging with pharmacological stress [61]. Radiation doses for CTMP can be expected to decrease further, as radiation doses $<1 \mathrm{mSv}$ on cCTA studies are still state of the art [61].

\subsection{Clinical setting}

As mentioned in the introduction of this chapter, CT myocardial perfusion offers additional functional data of the myocardial blood supply. In contrast, ordinary cCTA only provides anatomical evaluation of the heart. Combined cCTA plus CTMP provides incremental diagnostic value compared with CCTA alone to assess the status of the myocardial blood supply and for the detection of significant coronary stenosis $[6,57,58]$. 
Compared with other functional noninvasive methods such as single photon emission computed tomography (SPECT) or cardiac magnetic resonance perfusion imaging (cMRI), CTMP is a recent technology.

SPECT is a nuclear imaging technique with tracer substances, such as thallium-201 or technetium-99. Myocardial enhancement of this tracer differs in damaged myocardium. A rotating gamma camera enables three-dimensional tomographic reconstruction [6]. According to current guidelines of the American Heart Association and American College of Cardiology, SPECT is used for the diagnosis of CAD, risk stratification, myocardial viability, and left ventricular function [62]. Rest and stress SPECT acquisitions allow evaluation of ischemic reversibility.

Cardiac magnetic resonance imaging (cMRI) offers anatomical information and a variety of functional aspects, such as assessment of myocardial perfusion during rest and stress acquisition and myocardial viability. SPECT has lower temporal and spatial resolution than cMRI [6]. The large CE-MARC trial led to higher sensitivity with cMRI than with SPECT and postulated cost-effectiveness and more use of this method [63, 64]. Patients with devices such as cardiac pacemakers or internal cardiac defibrillator (ICD) are often associated with great effort, regarding cMRI requirements. For patients with a tendency to claustrophobia, cMRI is potentially not the adequate examination due to long acquisition time [65]. On the other hand, cMRI is advantageous because of no ionizing radiation.

CT myocardial perfusion or other functional techniques are not reasonable in each clinical question compared to ordinary cCTA for ruling out CAD. In a situation of acute chest pain in a patient with low pretest probability of $\mathrm{CAD}$, an extensive stress examination (irrespective of the imaging technique) is potentially not indicated due to prolonged examination. The availability in case of short-term request of such a comprehensive examination represents a further doubtful aspect in the clinical setting. However, CT myocardial perfusion has the potential to overcome these obstacles.

\section{Conclusion and further perspective}

Myocardial perfusion derived from computed tomography is a growing diagnostic method that provides a comprehensive evaluation of coronary artery disease along with functional assessment of the myocardium with promising findings in current clinical studies. Combining cCTA with CTMP significantly improves specificity and positive predictive value $[57,58]$.

The multicentre DECIDE-Gold trial [66] might contribute in establishment myocardial perfusion within the clinical setting. Focus of current research is, e.g., the order and general need of all three sequences in times of modern dual energy computed tomography scanners. Meinel et al. postulates a dual energy rest-stress approach as protocol of choice. Furthermore, he achieves excellent sensitivity and specificity in a rest-only approach [67]. This would represent substantial advantage for the patient. Functional situation of myocardial blood supply could be derived simultaneously from ordinary coronary computed tomography angiography within the same examination, without additional radiation, drugs or prolonged examination. 
CT myocardial perfusion imaging offers great potential to reclassify findings in CCTA and to evaluate the myocardial blood supply [68]. Regarding risk of invasive coronary angiography [69], an initial noninvasive diagnostic selection would be desirable to reduce invasive angiograms, showing no obstructive CAD. Addition of CTMP to cCTA holds highly promising potential to adopt this role and to establish $\mathrm{CT}$ as a single imaging examination for comprehensive evaluation of CAD and direct assessment of myocardial ischemia in one examination (Figure 1).

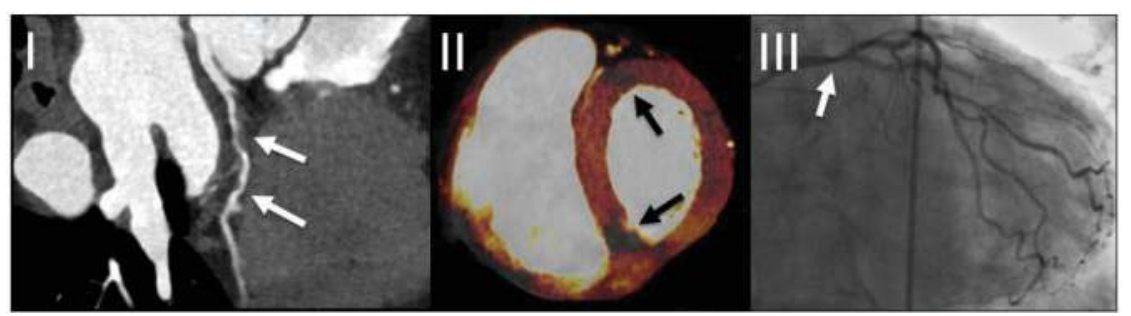

Figure 1.59-year-old female with known hypertension presenting with chest pain. (I) cCTA show several moderate stenoses of the LAD (arrows). (II) DECT show minor iodine distribution within basal LAD and RCA territory as a sign of hemodynamic significance (arrows). (III) Invasive catheter angiography show severe artery disease of all three vessels. Subtotal stenosis of RCA, significant stenosis of the left main trunk (arrow) and 75\% stenosis of mid RCX and Ramus marginalis. cCTA, coronary computed tomography; DECT, dual-energy computed tomography; LAD, left anterior descending; RCA, right coronary artery; RCX, ramus circumflexus.

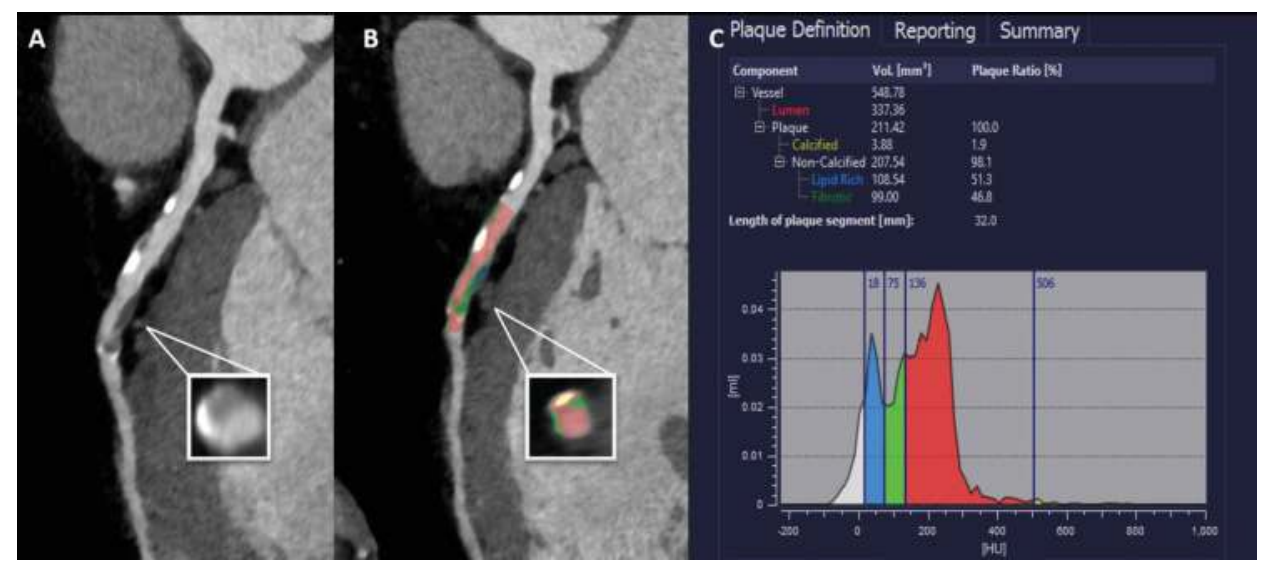

Figure 2.(A) cCTA shows stenotic noncalcified plaque of the LAD. (B + C) Color-coded automated plaque quantification by the analysis software showed the plaque composition as predominantly noncalcified. cCTA, coronary computed tomography angiography; LAD, left anterior descending.

\section{CT-FFR}

The invasive measurement of the fractional flow reserve is currently the accepted reference standard to determine, whether a coronary stenosis is hemodynamically relevant and is 
therefore implemented in the guidelines [70]. The FAME study has proved that FFR guided coronary revascularization is associated with reduced rates of death, myocardial infarction or target vessel revascularization [71]. In clinical routine, the use of invasive FFR is associated with risks and complications such a severe bleeding, arrhythmia, stroke, and coronary dissections depending on the experience of the interventional cardiologist [72].

Novel technologies have been developed to calculate noninvasive FFR from routine cCTA datasets using computational fluid dynamics. The main advantage of this technology is the markly improvement of specificity and positive predictive value compared to standard cCTA, without additional stress medication, image protocols, and radiation exposure (Table 5). While the first studies concentrated on the general feasibility and diagnostic performance, further clinical studies validated the cost-effectiveness. The PLATFORM-study showed that the numbers of patients without anatomically obstructive CAD $(p<0.0001)$ could be significantly improved with the CT-FFR arm, while the secondary endpoint radiation exposure showed no difference (9.9 vs. $9.4 \mathrm{mSv}, p=0.20)$ [73].

There are the first head-to-head comparisons of CT-FFR compared stress CT myocardial perfusion (CTP) in patients with CAD with a per-vessel specificity of was $66 \%$ for cCTA, $77 \%$ for CT-FFR, and 91\% for CTP, respectively, while the diagnostic performance of cCTA alone

\begin{tabular}{|c|c|c|c|c|c|}
\hline & $\begin{array}{l}\text { Koo et al. } \\
\text { (DISCOVER- } \\
\text { FLOW) [77] }\end{array}$ & $\begin{array}{l}\text { Min et al. } \\
\text { (DeFACTO) [78] }\end{array}$ & $\begin{array}{l}\text { Nørgaard et al. } \\
\text { (NXT-Trial) [79] }\end{array}$ & Renker et al. [80] & Coenen et al. [81] \\
\hline Vessels & 159 & 407 & 484 & 67 & 189 \\
\hline $\begin{array}{l}\text { Vessels with } \\
\text { intermediate } \\
\text { stenosis } \\
(30-70 \%)\end{array}$ & $66 / 159$ [25] (41.5\%) & $\begin{array}{l}150 / 407[26] \\
(36.9 \%)\end{array}$ & $235 / 484(48.6 \%)$ & $39 / 67(58.2 \%)$ & $144 / 189(76.2 \%)$ \\
\hline Sensitivity (\%) & $\begin{array}{l}87.9(76.7-95.0) \\
{[91.4(81.0-97.1)]}\end{array}$ & 80 (73-86) [N.A.] & $\begin{array}{l}84(75-89)[83 \\
(74-89)]\end{array}$ & $\begin{array}{l}85(62-97)[90 \\
(68-98)]\end{array}$ & $\begin{array}{l}87.5(78.2-93.8) \\
{[81.3(71.0-89.1)]}\end{array}$ \\
\hline Specificity (\%) & $\begin{array}{l}82.2(73.3-89.1) \\
{[39.6(30.0-49.8)]}\end{array}$ & 61 (54-67) [N.A.] & $\begin{array}{l}86(82-89)[60 \\
(56-65)]\end{array}$ & $\begin{array}{l}85(72-94)[34 \\
(21-49)]\end{array}$ & $\begin{array}{l}65.1(55.4-74.0] \\
{[37.6(28.5-47.4)]}\end{array}$ \\
\hline PPV (\%) & $\begin{array}{l}73.9(61.9-83.7) \\
{[46.5(37.1-56.1)]}\end{array}$ & 56 (49-62) [N.A.] & $\begin{array}{l}61(53-69)[33 \\
(27-39)]\end{array}$ & $\begin{array}{l}71(49-87)[37 \\
(23-52)]\end{array}$ & $\begin{array}{l}64.8(55.0-73.8)] \\
{[48.9(40.1-57.7)]}\end{array}$ \\
\hline NPV (\%) & $\begin{array}{l}92.2(84.6-96.8] \\
{[88.9(75.9-96.3)]}\end{array}$ & 84 (78-89) [N.A.] & $\begin{array}{l}95(93-97)[92 \\
(88-95)]\end{array}$ & $\begin{array}{l}93(81-98)[89 \\
(65-98)]\end{array}$ & $\begin{array}{l}87.7(78.5-93.9) \\
{[73.2(59.7-84.2)]}\end{array}$ \\
\hline Accuracy (\%) & $\begin{array}{l}84.3(77.7-90.0) \\
{[58.5(50.4-66.2)]}\end{array}$ & N.A. [N.A.] & $\begin{array}{l}86(83-89)[65 \\
(61-69)]\end{array}$ & N.A. [N.A.] & $\begin{array}{l}74.6(68.4-80.8) \\
{[56.1(49.0-63.2)]}\end{array}$ \\
\hline AUC & $\begin{array}{l}0.90 \text { (N.A.) }[0.75 \\
\text { (N.A.)] }(p=0.001)\end{array}$ & N.A. [N.A.] & $\begin{array}{l}0.93(0.91-0.95) \\
{[0.79(0.74-0.84)]} \\
(p<0.001)\end{array}$ & $\begin{array}{l}0.92 \text { (N.A.) } \\
{[0.72 \text { (N.A.) }](p} \\
<0.005)\end{array}$ & $\begin{array}{l}0.83 \text { (N.A.) }[0.64 \\
\text { (N.A.)] }(\mathrm{p}<0.001)\end{array}$ \\
\hline
\end{tabular}

CT-FFR $<0.80$ (95\% CI) und cCTA stenosis $\geq 50 \%$ (95\% CI) [in brackets] were defined as cut-off values. AUC, area under the curve; cCTA, coronary CT-angiography; CT-FFR, CT-based FFR; FFR, Fractional flow reserve; N.A., not available; NPV, negative predictive value; PPV, positive predictive value.

Table 5. Diagnostic accuracy of CT-FFR and CCTA compared to invasive FFR as the reference standard on a per vessel $(\mathrm{n}=1306)$ basis. 
was significantly improved by combination with CT-FFR or CTP [74]. Meta-analysis shows that CT-FFR can act in the context of other myocardial perfusion modalities as a potential gatekeeper for invasive revascularization (Table 6) in patients with suspected or known CAD using invasive FFR as the reference standard [75]. Due to time-consuming off-site calculation and transfer of the datasets to external core laboratory the clinical impact is limited. Thus, a novel solution for physician-driven CT-FFR derivation using regular on-site workstations was developed. This CT-FFR algorithm applies reduced-order models for more expeditious calculation, but is currently not commercially available [76].

\begin{tabular}{llllll}
\hline & CT-FFR & CT-perfusion & SPECT & PET & MRT \\
\hline $\begin{array}{l}\text { Number of } \\
\text { vessels }\end{array}$ & 714 & 1074 & 924 & 870 & 1830 \\
Sensitivity (\%) & $0.83(0.79-0.87)$ & $0.78(0.72-0.82)$ & $0.61(0.56-0.66)$ & $0.83(0.77-0.88)$ & $0.87(0.84-0.90)$ \\
Specificity (\%) & $0.77(0.74-0.80)$ & $0.86(0.83-0.88)$ & $0.84(0.81-0.87)$ & $0.89(0.86-0.91)$ & $0.91(0.89-0.92)$ \\
PLR & $3.76(2.17-6.54)$ & $5.74(3.48-9.46)$ & $3.76(2.74-5.16)$ & $7.43(5.03-10.99)$ & $8.27(4.93-13.87)$ \\
NLR & $0.23(0.16-0.35)$ & $0.22(0.12-0.39)$ & $0.47(0.37-0.59)$ & $0.15(0.05-0.44)$ & $0.16(0.13-0.21)$ \\
AUC & N.A. & $0.91(0.86-0.96)$ & $0.83(0.67-0.98)$ & $0.95(0.91-0.99)$ & $0.95(0.93-0.97)$ \\
\hline
\end{tabular}

Adapted from von Gonzalez et al. [82] and Takx et al. [75]. AUC, area under the curve; cCTA, coronary CT-angiography; CT-FFR, CT based fractional flow reserve; FFR, fractional flow reserve; N.A., not available; NLR, negative likelihood ratio; PLR, positive likelihood ratio.

Table 6. Diagnostic accuracy of CT-FFR and other non-invasive modalities compared to invasive FFR.

Currently, CT-FFR is an interesting and sophisticated approach to identify functionally significant CAD in a noninvasive way. However, this promising technique is still in development and searching for its clinical application, and further evidence studies are necessary before CT-FFR is implemented for clinical use.

\section{Author details}

Stefan Baumann*, Philipp Kryeziu, Marlon Rutsch and Dirk Lossnitzer

*Address all correspondence to: stefanbaumann@gmx.at

First Department of Medicine-Cardiology, University Medical Centre Mannheim, Mannheim, Germany

\section{References}

[1] Boyd, D.P. and M.J. Lipton, Cardiac Computed Tomography. Proceedings of the IEEE, 1983. 71(3): pp. 298-307. 
[2] Rubin, G.D., et al., CT angiography after 20 years: a transformation in cardiovascular disease characterization continues to advance. Radiology, 2014. 271(3): pp. 633-52.

[3] Apfaltrer, P., et al., Coronary computed tomography - present status and future directions. Int J Clin Pract Suppl, 2011(173): pp. 3-13.

[4] Halliburton, S., et al., State-of-the-art in CT hardware and scan modes for cardiovascular CT. J Cardiovasc Comput Tomogr, 2012. 6(3): pp. 154-63.

[5] Heydari, B., et al., Diagnostic performance of high-definition coronary computed tomography angiography performed with multiple radiation dose reduction strategies. Can J Cardiol, 2011. 27(5): pp. 606-12.

[6] Bucher, A.M., et al., Cardiac CT for myocardial ischaemia detection and characterization--comparative analysis. Br J Radiol, 2014. 87(1043): p. 20140159.

[7] Douglas, P.S., et al., Outcomes of anatomical versus functional testing for coronary artery disease. N Engl J Med, 2015. 372(14): pp. 1291-300.

[8] Norgaard, B.L., et al., Coronary computed tomography angiography derived fractional flow reserve and plaque stress. Curr Cardiovasc Imaging Rep, 2016. 9: p. 2.

[9] Montalescot, G., et al., 2013 ESC guidelines on the management of stable coronary artery disease: the task force on the management of stable coronary artery disease of the European Society of Cardiology. Eur Heart J, 2013. 34(38): pp. 2949-3003.

[10] Wolk, M.J., et al., ACCF/AHA/ASE/ASNC/HFSA/HRS/SCAI/SCCT/SCMR/STS 2013 multimodality appropriate use criteria for the detection and risk assessment of stable ischemic heart disease: a report of the American College of Cardiology Foundation Appropriate Use Criteria Task Force, American Heart Association, American Society of Echocardiography, American Society of Nuclear Cardiology, Heart Failure Society of America, Heart Rhythm Society, Society for Cardiovascular Angiography and Interventions, Society of Cardiovascular Computed Tomography, Society for Cardiovascular Magnetic Resonance, and Society of Thoracic Surgeons. J Am Coll Cardiol, 2014. 63(4): pp. 380-406.

[11] Shaw, L.J., et al., Coronary computed tomographic angiography as a gatekeeper to invasive diagnostic and surgical procedures: results from the multicenter CONFIRM (Coronary CT angiography evaluation for clinical outcomes: an International multicenter) registry. J Am Coll Cardiol, 2012. 60(20): pp. 2103-14.

[12] Diamond, G.A. and J.S. Forrester, Analysis of probability as an aid in the clinical diagnosis of coronary-artery disease. N Engl J Med, 1979. 300(24): pp. 1350-8.

[13] Genders, T.S., et al., A clinical prediction rule for the diagnosis of coronary artery disease: validation, updating, and extension. Eur Heart J, 2011. 32(11): pp. 1316-30.

[14] Cerqueira, M.D., et al., Standardized myocardial segmentation and nomenclature for tomographic imaging of the heart. A statement for healthcare professionals from the Cardiac Imaging Committee of the Council on Clinical Cardiology of the American Heart Association. Circulation, 2002. 105(4): pp. 539-42. 
[15] Schmermund, A., et al., Non-invasive assessment of coronary Palmaz-Schatz stents by contrast enhanced electron beam computed tomography. Eur Heart J, 1996. 17(10): pp. 1546-53.

[16] Sun, Z. and A.M. Almutairi, Diagnostic accuracy of 64 multislice CT angiography in the assessment of coronary in-stent restenosis: a meta-analysis. Eur J Radiol, 2010. 73(2): pp. 266-73.

[17] Kumbhani, D.J., et al., Meta-analysis of diagnostic efficacy of 64-slice computed tomography in the evaluation of coronary in-stent restenosis. Am J Cardiol, 2009. 103(12): pp. 1675-81.

[18] Malagutti, P., et al., Use of 64-slice CT in symptomatic patients after coronary bypass surgery: evaluation of grafts and coronary arteries. Eur Heart J, 2007. 28(15): pp. 1879-85.

[19] Engelmann, M.G., et al., Non-invasive coronary bypass graft imaging after multivessel revascularisation. Int J Cardiol, 2000. 76(1): pp. 65-74.

[20] Frazier, A.A., et al., Coronary artery bypass grafts: assessment with multidetector CT in the early and late postoperative settings. Radiographics, 2005. 25(4): pp. 881-96.

[21] Budoff, M.J., et al., Diagnostic performance of 64-multidetector row coronary computed tomographic angiography for evaluation of coronary artery stenosis in individuals without known coronary artery disease: results from the prospective multicenter ACCURACY (Assessment by Coronary Computed Tomographic Angiography of Individuals Undergoing Invasive Coronary Angiography) trial. J Am Coll Cardiol, 2008. 52(21): pp. 1724-32.

[22] Min, J.K., L.J. Shaw, and D.S. Berman, The present state of coronary computed tomography angiography a process in evolution. J Am Coll Cardiol, 2010. 55(10): pp. 957-65.

[23] Sarwar, A., et al., Calcified plaque: measurement of area at thin-section flat-panel CT and 64-section multidetector CT and comparison with histopathologic findings. Radiology, 2008. 249(1): pp. 301-6.

[24] Hoffmann, U., et al., Predictive value of 16-slice multidetector spiral computed tomography to detect significant obstructive coronary artery disease in patients at high risk for coronary artery disease: patient-versus segment-based analysis. Circulation, 2004. 110(17): pp. 2638-43.

[25] Morin, R.L., T.C. Gerber, and C.H. McCollough, Radiation dose in computed tomography of the heart. Circulation, 2003. 107(6): pp. 917-22.

[26] Sun, Z. and K.H. Ng, Multislice CT angiography in cardiac imaging. Part III: radiation risk and dose reduction. Singapore Med J, 2010. 51(5): pp. 374-80.

[27] Peebles, C., Computed tomographic coronary angiography: how many slices do you need? Heart, 2006. 92(5): pp. 582-4.

[28] Brenner, D.J. and E.J. Hall, Computed tomography - an increasing source of radiation exposure. N Engl J Med, 2007. 357(22): pp. 2277-84.

[29] Earls, J.P., et al., Prospectively gated transverse coronary CT angiography versus retrospectively gated helical technique: improved image quality and reduced radiation dose. Radiology, 2008. 246(3): pp. 742-53. 
[30] Hausleiter, J. and T. Meyer, Tips to minimize radiation exposure. J Cardiovasc Comput Tomogr, 2008. 2(5): pp. 325-7.

[31] Halliburton, S.S., et al., SCCT guidelines on radiation dose and dose-optimization strategies in cardiovascular CT. J Cardiovasc Comput Tomogr, 2011. 5(4): pp. 198-224.

[32] Menke, J., et al., Head-to-head comparison of prospectively triggered vs retrospectively gated coronary computed tomography angiography: Meta-analysis of diagnostic accuracy, image quality, and radiation dose. Am Heart J, 2013. 165(2): pp. 154-63 e3.

[33] Linsen, P.V., et al., Computed tomography angiography with a 192-slice dual-source computed tomography system: improvements in image quality and radiation dose. J Clin Imaging Sci, 2016. 6: p. 44.

[34] Sun, K., et al., Prospectively electrocardiogram-gated high-pitch spiral acquisition mode dualsource CT coronary angiography in patients with high heart rates: comparison with retrospective electrocardiogram-gated spiral acquisition mode. Korean J Radiol, 2012. 13(6): pp. 684-93.

[35] Achenbach, S., et al., Coronary computed tomography angiography with a consistent dose below $1 \mathrm{mSv}$ using prospectively electrocardiogram-triggered high-pitch spiral acquisition. Eur Heart J, 2010. 31(3): pp. 340-6.

[36] Chen, M.Y., S.M. Shanbhag, and A.E. Arai, Submillisievert median radiation dose for coronary angiography with a second-generation 320-detector row CT scanner in 107 consecutive patients. Radiology, 2013. 267(1): pp. 76-85.

[37] Hounsfield, G.N., Computerized transverse axial scanning (tomography). 1. Description of system. Br J Radiol, 1973. 46(552): pp. 1016-22.

[38] Geyer, L.L., et al., State of the art: iterative CT reconstruction techniques. Radiology, 2015. 276(2): pp. 339-57.

[39] Renker, M., et al., Iterative image reconstruction techniques: applications for cardiac CT. J Cardiovasc Comput Tomogr, 2011. 5(4): pp. 225-30.

[40] Hou, Y., et al., The optimal dose reduction level using iterative reconstruction with prospective ECG-triggered coronary CTA using 256-slice MDCT. Eur J Radiol, 2012. 81(12): pp. 3905-11.

[41] Pontone, G., et al., Feasibility and diagnostic accuracy of a low radiation exposure protocol for prospective ECG-triggering coronary MDCT angiography. Clin Radiol, 2012. 67(3): pp. 207-15.

[42] Takx, R.A., et al., Coronary CT angiography: comparison of a novel iterative reconstruction with filtered back projection for reconstruction of low-dose CT-Initial experience. Eur J Radiol, 2013. 82(2): pp. 275-80.

[43] Leeson, M.D., et al., Atheromatous extracranial carotid arteries: CT evaluation correlated with arteriography and pathologic examination. Radiology, 1985. 156(2): pp. 397-402.

[44] Baumann, S., et al., Computed tomography imaging of coronary artery plaque: characterization and prognosis. Radiol Clin North Am, 2015. 53(2): pp. 307-15. 
[45] Fischer, C., et al., Coronary CT angiography versus intravascular ultrasound for estimation of coronary stenosis and atherosclerotic plaque burden: a meta-analysis. J Cardiovasc Comput Tomogr, 2013. 7(4): pp. 256-66.

[46] Achenbach, S., et al., Detection of calcified and noncalcified coronary atherosclerotic plaque by contrast-enhanced, submillimeter multidetector spiral computed tomography: a segment-based comparison with intravascular ultrasound. Circulation, 2004. 109(1): pp. 14-7.

[47] Nakazato, R., et al., Quantification and characterisation of coronary artery plaque volume and adverse plaque features by coronary computed tomographic angiography: a direct comparison to intravascular ultrasound. Eur Radiol, 2013. 23(8): pp. 2109-17.

[48] Finn, A.V., et al., Concept of vulnerable/unstable plaque. Arterioscler Thromb Vasc Biol, 2010. 30(7): pp. 1282-92.

[49] Muller, J.E., G.H. Tofler, and P.H. Stone, Circadian variation and triggers of onset of acute cardiovascular disease. Circulation, 1989. 79(4): pp. 733-43.

[50] Virmani, R., et al., Lessons from sudden coronary death: a comprehensive morphological classification scheme for atherosclerotic lesions. Arterioscler Thromb Vasc Biol, 2000. 20(5): pp. 1262-75.

[51] Stone, G.W., et al., A prospective natural-history study of coronary atherosclerosis. N Engl J Med, 2011. 364(3): pp. 226-35.

[52] Tesche, C., et al., Prognostic implications of coronary CT angiography-derived quantitative markers for the prediction of major adverse cardiac events. J Cardiovasc Comput Tomogr, 2016. 10(6): pp. 458-465.

[53] Cavalcante, R., et al., Validation of coronary computed tomography angiography scores for noninvasive assessment of atherosclerotic burden through a comparison with multivessel intravascular ultrasound. Atherosclerosis, 2016. 247: pp. 21-7.

[54] Gorenoi, V., M.P. Schonermark, and A. Hagen, CT coronary angiography vs. invasive coronary angiography in CHD. GMS Health Technol Assess, 2012. 8: p. Doc02.

[55] Jacobs, J.E., et al., ACR practice guideline for the performance and interpretation of cardiac computed tomography (CT). J Am Coll Radiol, 2006. 3(9): pp. 677-85.

[56] Kristensen, T.S., et al., Correlation between coronary computed tomographic angiography and fractional flow reserve. Int J Cardiol, 2010. 144(2): pp. 200-5.

[57] Sorgaard, M.H., et al., Diagnostic accuracy of static CT perfusion for the detection of myocardial ischemia. A systematic review and meta-analysis. J Cardiovasc Comput Tomogr, 2016.

[58] Caruso, D., et al., Dynamic CT myocardial perfusion imaging. Eur J Radiol, 2016. 85(10): pp. 1893-9.

[59] Ambrose, M.S., et al., CT perfusion: ready for prime time. Curr Cardiol Rep, 2011. 13(1): pp. 57-66.

[60] Pelgrim, G.J., et al., The dream of a one-stop-shop: meta-analysis on myocardial perfusion CT. Eur J Radiol, 2015. 84(12): pp. 2411-20. 
[61] Feuchtner, G., et al., Adenosine stress high-pitch 128-slice dual-source myocardial computed tomography perfusion for imaging of reversible myocardial ischemia: comparison with magnetic resonance imaging. Circ Cardiovasc Imaging, 2011. 4(5): pp. 540-9.

[62] Klocke, F.J., et al., ACC/AHA/ASNC guidelines for the clinical use of cardiac radionuclide imaging-executive summary. J Am Coll Cardiol, 2003. 42(7): pp. 1318-1333.

[63] Greenwood, J.P., et al., Comparison of cardiovascular magnetic resonance and single-photon emission computed tomography in women with suspected coronary artery disease from the Clinical Evaluation of Magnetic Resonance Imaging in Coronary Heart Disease (CE-MARC) Trial. Circulation, 2014. 129(10): pp. 1129-38.

[64] Walker, S., et al., Cost-effectiveness of cardiovascular magnetic resonance in the diagnosis of coronary heart disease: an economic evaluation using data from the CE-MARC study. Heart, 2013. 99(12): pp. 873-81.

[65] Bluemke, D.A., et al., Noninvasive coronary artery imaging: magnetic resonance angiography and multidetector computed tomography angiography: a scientific statement from the american heart association committee on cardiovascular imaging and intervention of the council on cardiovascular radiology and intervention, and the councils on clinical cardiology and cardiovascular disease in the young. Circulation, 2008. 118(5): pp. 586-606.

[66] Truong, Q.A., et al., Rationale and design of the dual-energy computed tomography for ischemia determination compared to "gold standard" non-invasive and invasive techniques (DECIDEGold): a multicenter international efficacy diagnostic study of rest-stress dual-energy computed tomography angiography with perfusion. J Nucl Cardiol, 2015. 22(5): pp. 1031-40.

[67] Meinel, F.G., et al., First-arterial-pass dual-energy CT for assessment of myocardial blood supply: do we need rest, stress, and delayed acquisition? Comparison with SPECT. Radiology, 2014. 270(3): pp. 708-16.

[68] Osawa, K., et al., Diagnostic performance of first-pass myocardial perfusion imaging without stress with computed tomography (CT) compared with coronary CT angiography alone, with fractional flow reserve as the reference standard. PLoS One, 2016. 11(2): p. e0149170.

[69] Noto, T.J., Jr., et al., Cardiac catheterization 1990: a report of the registry of the Society for Cardiac Angiography and Interventions (SCAEI). Cathet Cardiovasc Diagn, 1991. 24(2): pp. 75-83.

[70] Authors/Task Force, m., et al., 2014 ESC/EACTS Guidelines on myocardial revascularization: the task force on myocardial revascularization of the European Society of Cardiology (ESC) and the European Association for Cardio-Thoracic Surgery (EACTS)developed with the special contribution of the European Association of Percutaneous Cardiovascular Interventions (EAPCI). Eur Heart J, 2014. 35(37): pp. 2541-619.

[71] Tonino, P.A., et al., Fractional flow reserve versus angiography for guiding percutaneous coronary intervention. N Engl J Med, 2009. 360(3): pp. 213-24.

[72] Park, S.J., et al., Trends in the outcomes of percutaneous coronary intervention with the routine incorporation of fractional flow reserve in real practice. Eur Heart J, 2013. 34(43): pp. 3353-61. 
[73] Douglas, P.S., et al., Clinical outcomes of fractional flow reserve by computed tomographic angiography-guided diagnostic strategies vs. usual care in patients with suspected coronary artery disease: the prospective longitudinal trial of FFR(CT): outcome and resource impacts study. Eur Heart J, 2015. 36(47): pp. 3359-67.

[74] Yang, D.H., et al., Diagnostic performance of on-site CT-derived fractional flow reserve versus CT perfusion. Eur Heart J Cardiovasc Imaging, 2016. 35: pp. 1120-30.

[75] Takx, R.A., et al., Diagnostic accuracy of stress myocardial perfusion imaging compared to invasive coronary angiography with fractional flow reserve meta-analysis. Circ Cardiovasc Imaging, 2015. 8(1).

[76] Baumann, S., et al., Different approaches for coronary computed tomography angiographyderived versus invasive fractional flow reserve assessment. Am J Cardiol, 2016. 117(3): p. 486.

[77] Koo, B.K., et al., Diagnosis of ischemia-causing coronary stenoses by noninvasive fractional flow reserve computed from coronary computed tomographic angiograms. Results from the prospective multicenter DISCOVER-FLOW (Diagnosis of Ischemia-Causing Stenoses Obtained Via Noninvasive Fractional Flow Reserve) study. J Am Coll Cardiol, 2011. 58(19): pp. 1989-97.

[78] Min, J.K., et al., Diagnostic accuracy of fractional flow reserve from anatomic CT angiography. Jama, 2012. 308(12): pp. 1237-45.

[79] Norgaard, B.L., et al., Diagnostic performance of noninvasive fractional flow reserve derived from coronary computed tomography angiography in suspected coronary artery disease: the NXT trial (Analysis of Coronary Blood Flow Using CT Angiography: Next Steps). J Am Coll Cardiol, 2014. 63(12): pp. 1145-55.

[80] Renker, M., et al., Comparison of diagnostic value of a novel noninvasive coronary computed tomography angiography method versus standard coronary angiography for assessing fractional flow reserve. Am J Cardiol, 2014. 114(9): pp. 1303-8.

[81] Coenen, A., et al., Fractional flow reserve computed from noninvasive CT angiography data: diagnostic performance of an on-site clinician-operated computational fluid dynamics algorithm. Radiology, 2015. 274(3): pp. 674-83.

[82] Gonzalez, J.A., et al., Meta-Analysis of diagnostic performance of coronary computed tomography angiography, computed tomography perfusion, and computed tomography-fractional flow reserve in functional myocardial ischemia assessment versus invasive fractional flow reserve. Am J Cardiol, 2015. 116(9): pp. 1469-78. 